\title{
Um Índice para Avaliação da Variabilidade Espaço-Temporal das Chuvas no Brasil
}

\author{
Raquel Fetter ${ }^{1}$ iD , Carlos Henke de Oliveira ${ }^{2}$, Ercília Torres Steinke ${ }^{3}$ \\ ${ }^{1}$ Programa de Pós-Graduação em Geografia, Departamento de Geografia, \\ Instituto de Ciências Humanas, Universidade de Brasília, \\ Campus Darcy Ribeiro, Brasília, DF, Brasil. \\ ${ }^{2}$ Laboratório de Ecologia Aplicada, Departamento de Ecologia, \\ Instituto de Ciências Biológicas, Universidade de Brasilia, \\ Campus Darcy Ribeiro, Brasília, DF, Brasil. \\ ${ }^{3}$ Laboratório de Climatologia Geográfica, Departamento de Geografia, \\ Instituto de Ciências Humanas, Universidade de Brasília, \\ Campus Darcy Ribeiro, Brasília, DF, Brasil.
}

Received: August 26, 2017 - Accepted: January 25, 2018

\begin{abstract}
Resumo
O Brasil apresenta elevada variabilidade espaço-temporal pluviométrica, sendo fundamental representá-la sistematicamente visando conhecer seus efeitos sobre o ciclo hidrológico e as diversas atividades humanas. Assim, é proposto o "Índice de Proporção de Escalas" (IPE), com o objetivo de avaliar a contribuição relativa da variação da quantidade de chuvas em função da maior/menor estruturação espaço/temporal das mesmas. Foram obtidos valores do índice para 809 localizações no país, a partir dos quais foram identificados cinco padrões principais de IPE com distintas configurações espaço-temporal. Foi observado que, em três regiões de fronteira próximas aos limites territoriais do Brasil correspondem a uma faixa de baixos IPEs (fator espacial relativamente mais importante). Enquanto na região central e no extremo sul do país, prevalecem altos IPEs (fator temporal relativamente mais importante). Com isso, a partir dos padrões descritos acima foi possível relacioná-los aos sistemas formadores do tempo meteorológico e, conduzir a uma caracterização preliminar destas regiões que auxiliam no entendimento da variabilidade espaço-temporal das chuvas.
\end{abstract}

Palavras-chave: chuva, variabilidade espacial, variabilidade temporal, Índice de Proporção de Escalas.

\section{Proposition of an Index for the Study of the Variability of Space-Temporal Rainfall in Brazil}

\begin{abstract}
Brazil shows a high spatial and temporal pluviometric variability. Therefore, it is crucial to systematically represent this phenomena in order to know the influence of precipitation for the hydrological cycle and several human activities. Thus, it is proposed a metrics called IPE (Index of Scale Proportion) to assess the relative contribution of the time and space on the rainfall variability, aiming to identify multi-scalar climatic patterns and to relate them to the main meteorological driver systems. We used data from 809 locations and identified five main patterns for IPE in distinct spatiotemporal configuration. The patterns called A, B and C describe a range of low IPE values (major importance of space relative to time) covering a large circular area with its limits near to the country edge. The patterns called $\mathrm{D}$ and $\mathrm{E}$ are located respectively on central and south area of country, showing high IPE values (time factor was relatively more important). It was possible to correlate the described IPE patterns to the well-know weather systems, gathering a preliminary characterization that help us to clarify the spatiotemporal rainfall variability.
\end{abstract}

Keywords: rain, spatial variability, temporal variability, scale, index.

Autor de correspondência: Raquel Fetter, raquelfetter@yahoo.com.br. 


\section{Introdução}

A representação da distribuição das chuvas tanto no espaço como no tempo é essencial para determinar a influência da precipitação no ciclo hidrológico e em diversas atividades humanas (Koutsoyiannis, 2006). No entanto, mensurar as inúmeras variáveis que determinam a variabilidade em diferentes escalas de tempo e de espaço é uma tarefa difícil diante da complexidade inerente aos processos (Barros e Lettenmaier, 1994; Harris e Georgiou, 2001) e principalmente, que ao migrar para escalas de maior detalhe identifica-se um maior número de fatores e de interações que modulam e influenciam a precipitação.

Há diversos estudos que focam no efeito das feições geográficas sobre o clima, enquanto outros apontam para as oscilações e mudanças nas séries temporais. Tais iniciativas convergem para a questão central do tempo e/ou do espaço como determinantes das variações contida nos dados.

Dentre os estudos do clima brasileiro que caracterizam as feições geográficas destaca-se o de Soares et al. (2014), que conclui que a distribuição espacial da precipitação anual, sazonal e mensal, na região hidrográfica da Bahia da Ilha Grande-RJ, é fortemente influenciada pela topografia e pela distância do litoral. Já o trabalho de Lyra et al. (2014) identificaram a intensificação das brisas marinhas pelos ventos alísios do sudeste, no período seco, os quais, submetidos a características orográficas causam chuvas nas maiores altitudes e a barlavento da Serra da Borborema, a qual atua como barreira da umidade nas regiões áridas. Gomes-Filho (1979) sugere que o alto albedo do semiárido nordestino gera uma coluna atmosférica mais fria sobre a região, induzindo a subsidência do ar com a consequente inibição da convecção e da precipitação, principalmente no período em que o anticiclone do Atlântico Sul está mais próximo à região. No período chuvoso, no estado de Rondônia, Carey et al. (2001) encontraram totais mensais de precipitação mais elevados para as categorias de superfície compostas por áreas elevadas e florestadas quando comparadas as categorias de áreas planas e nãoflorestadas (agropecuária). Isso remete à ideia de um forte efeito do contexto local (uso da terra e geomorfologia) e consequentemente do espaço, sobre as chuvas. Para o mesmo período na Amazônia, Machado (2002) mostrou que os sistemas convectivos de mesoescala formam-se preferencialmente sobre regiões mais elevadas indicando a importância da topografia, mesmo que nestes locais as diferenças de elevação não são muito pronunciadas. Na região metropolitana de São Paulo, Vemado e Pereira Filho (2016) descreveram a combinação do efeito de ilha de calor local com as brisas marinhas em episódios intensos de chuva, os quais podem chegar a cerca de quatro vezes mais do que em áreas rurais ou menos urbanizadas no seu entorno.

Dentre os estudos que focam na estruturação temporal do fenômeno climático, Silva Dias et al. (2012) identificaram um elevado percentual de participação dos fenô- menos El Niño Oscilação Sul (ENSO), Oscilação Decadal do Pacífico, Oscilação do Atlântico Norte e a temperatura da superfície do mar na costa próxima de São Paulo, na frequência crescente de extremos pluviométricos durante a estação seca. No entanto, esses mesmos fenômenos explicam uma fração menor da variância total de eventos extremos, indicando a participação de outros fatores espaciais locais, tais como o crescimento da ilha de calor urbana e da poluição do ar. Hounsou-Gbo et al. (2015) avaliaram a contribuição das interações atmosfera-oceano Atlântico tropical sobre a variabilidade das chuvas no nordeste brasileiro. Loureiro et al. (2015) também identificaram a influência de anomalias meteorológicas dos oceanos Atlântico e Pacífico no comportamento heterogêneo da variabilidade espaço-temporal das chuvas na região hidrográfica Tocantins/Araguaia. Kayano e Andreoli (2009) analisaram a relação entre a temperatura da superfície do Atlântico Tropical com a variabilidade das chuvas no nordeste do Brasil, durante diferentes fases da Oscilação Multidecadal Atlântica.

Enquanto a maioria dos estudos foca num espaço geográfico mais restrito, outros visam amplas áreas, demandando maior volume de dados, por vezes de fontes distintas, e priorizando tecnologias mais robustas no gerenciamento de bancos de dados. Villar et al. (2009) analisaram a bacia amazônica num estudo que contemplou cinco países. No aspecto temporal, o estudo abordou a sazonalidade e a identificação de tendências. No aspecto espacial, os processos determinates da variabilidade versaram tanto sobre características locais, como a topografia, quanto caracterísicas de mesoescala, sinóticas e de acoplamento atmosfera-oceano.

No geral, os estudos citados até aqui trabalharam com séries temporais de unidades espaciais distintas, cujos diagnósticos permitem apenas a comparação entre as mesmas. Apesar dos dados serem explicitamente organizados no espaco e no tempo, geralmente há sistematização no tempo não havendo sistematização análoga para o espaço, por exemplo, pela organização de uma malha com resolução espacial constante. Um trabalho que mostrou notável avanço ao superar tais limitações é apresentado por Rysman et al. (2013), que avaliaram dados de chuvas em resolução temporal original de cinco minutos e espacial de $1 \mathrm{~km}^{2}$, com emprego de um radar meteorológico no sul da França. Apesar da limitação espacial na ordem de poucas centenas de km, o emprego da análise espectral no tempo e espaço possibilitou identificar a densidade (ou força) do espectro, conseguindo enquadrar os distintos eventos de chuva dentro dos sistemas de tempo conhecidos, tais como tempestades (até $20 \mathrm{~min}$ em $7 \mathrm{~km}$ ), sistemas convectivos (até 45 min em $20 \mathrm{~km}$ ), de mesoescala (até $3 \mathrm{~h}$ em $100 \mathrm{~km}$ ), sinóticos (alguns dias em milhares de quilômetros), etc.

De fato, as iniciativas que realmente integram espaço e tempo de forma explícita e sistematizada ainda são escassas, porém importantes para a identificação da parcela de contribuição dos processos ou mesmo na identificação de 
possíveis causas de mudanças no regime de chuvas. $\mathrm{Na}$ Suécia, Busuioc et al. (2001) constataram que nos meses de inverno a variabilidade pluviométrica é dominada pelo tempo, o que conduziu os estudos posteriores à identificação dos mecanismos responsáveis por alterações no regime da precipitação em determinados períodos da série histórica. Na Península de Delmarva, a leste da Virgínia e nordeste da Carolina do Norte (EUA), Tokay et al. (2014) identificaram que no período de verão ocorre alta variabilidade espacial a qual é menor nos meses de inverno. Além disso, as correlações entre a precipitação aumentam com períodos de integração mais longos, indicando baixa variabilidade temporal em ambas as estações. No Irã, Javari (2016) identificou menor efeito espacial na sazonalidade da precipitação do que temporal, e também que as séries temporais mensais e anuais são relativamente mais homogêneas em comparação com as séries sazonais.

O presente trabalho tem como objetivo avaliar a contribuição relativa da variabilidade espaço-temporal das chuvas no Brasil por meio de um Índice que se baseia na variação da quantidade de chuvas em função da maior/menor estruturação espacial/temporal das mesmas. Ao mesmo tempo em que tal iniciativa foi oportunizada pela estruturação de um robusto banco de dados articulado a scripts computacionais para análises geoestatísticas, considera-se a carência de estudos cujo escopo conceitual considere análises sistemáticas e integradoras dos fatores espaçotemporais e cujo escopo territorial considere todo o território brasileiro, com possível contribuição para políticas públicas nacionais. No tocante ao estado da arte, a breve revisão bibliográfica até aqui conduzida também alicerça nossa iniciativa principalmente ao notar a escassez de trabalhos com organização dos dados pautada numa malha sistemática, cuja análise não incorra em distorções ou tendenciosidades e com a possível identificação de padrões espaciais errôneos por mero artifício metodológico, vício nos dados ou seletividade por análise subjetiva.

Assim, o presente estudo mescla a oportunidade tecnológica a uma demanda por melhorias nas políticas nacionais que tangenciam o clima, a meteorologia e a tomada de decisões, porém fundamentada no princípio de que o tempo e espaço sejam efetivamente estudados de forma integrada.

\section{Material e Métodos}

\subsection{Estruturação do banco de dados}

A construção do índice baseou-se em registros pluviométricos diários para o Brasil, obtidos da Agência Nacional de Águas (ANA). O banco de dados foi estruturado em SQL e sua versão original contém aproximadamente 110 milhões de registros pluviométricos diários, numa série iniciada no ano 1888. Para o presente estudo, foi utilizado um subconjunto correspondendo a uma série temporal com poucas interrupções, coincidindo ao período entre 1975 e 2010 (36 anos), com dados de 1.727 estações meteoro- lógicas. Toda operação com dados foi realizada por scripts em linguagem PHP, dada a versatilidade na manipulação de dados estruturados em SQL. A série passou por análise de consistência, utilizando o método desenvolvido por Fetter et al. (2016). Foram eliminados os registros considerados inconsistentes, de acordo com os recordes históricos de chuvas no Brasil. Foram estabelecidos limites que caracterizaram os dados $\leq 100 \mathrm{~mm} / \mathrm{dia}(99,9 \%$ dos casos) como consistentes, $\geq$ de $350 \mathrm{~mm} / \mathrm{dia}(0,003 \%)$ como inconsistentes, e os valores $>100 \mathrm{~mm} /$ dia e $<350 \mathrm{~mm} /$ dia como suspeitos. Os registros suspeitos foram considerados inconsistentes quando diferiram mais de dez vezes da média dos registros das dez estações mais próximas, considerando que os mesmos erros não ocorreriam em estações diferentes, que o dado suspeito mesmo que correto deveria diferir das estações vizinhas, porém, essa diferença não deveria ser demasiadamente grande.

A krigagem (Krige, 1951) é um procedimento geoestatístico de interpolação espacial frequentemente conduzido em Sistemas de Informações Geográficas (SIG), que emprega um semivariograma construído para uma serie espacial de dados, e não para uma série temporal. O método de krigagem ordinária foi executado sobre uma grade sistemática de $1^{\circ} \times 1^{\circ}$ de arco de latitude e longitude que totalizaram 809 quadrantes $(\mathrm{Q})$ sobre o território brasileiro $\mathrm{e}$, para os quais, os registros diários foram generalizados para a resolução mensal $(\mathrm{M})$. Assim, o número de krigagens demandada para atingir a resolução mensal foi de 432 (12 meses + 36 anos), número demasiadamente elevado para ser processado manualmente em ambiente SIG, daí o emprego de scripts computacionais para a automação no presente estudo.

A krigagem consiste em fornecer estimadores não tendenciosos e variância mínima para o valor de um atributo, em uma posição não amostrada, a partir de um conjunto de amostras vizinhas, levando-se em consideração, a estrutura de variação espacial (Krige, 1951), o que pressupõe a existência de correlação entre os dados (Isaaks e Srivastava, 1989). O método se destaca dentre os demais por apresentar resultados mais realistas na manipulação de dados pluviométricos (Reinstorf et al., 2005). Um dos principais motivos do seu destaque, segundo Bargaoui e Chebbi (2009) é a construção do semivariograma para expressar à dependência espacial através da medida da variância dos valores amostrais, separados por uma distância $h$ (Eq. (1)). A formulação matemática aqui empregada tem base em Camargo (1998).

$$
y(h) \frac{1}{2 N(h)}=\sum_{i=1}^{N(h)}[Z(x i)-Z(x i+h)]^{2}
$$

onde $N(h)=$ número de pares de valores amostrados [ $Z(x i)$ $Z(x i+h)]$ separados pelo vetor $h$.

Verificada a dependência espacial, a krigagem utiliza as informações do semivariograma para encontrar pesos a 
serem associados às amostras com valores conhecidos, para estimar o valor de pluviosidade para os pontos desconhecidos, nesse caso, o centro dos quadrantes. A estimativa por krigagem ordinária busca por um conjunto de ponderadores que minimize a variância do erro da estimativa (Landim, 2006) (Eq. (2))

$$
K \cdot \lambda=j \rightarrow \lambda=k \cdot K^{-1}
$$

onde $K$ e $\lambda$ são matrizes das covariâncias e $k$ o vetor dos pesos. Os pesos são atribuídos arbitrariamente (Eq. (3)).

$$
Z(x i)=f(x i)+e(x i), \quad n=1,2, \ldots, n
$$

onde $f(x i)=$ função aleatória $F$ num ponto $x i$; $(x i)$ são realizações médias de zero e erros.

A correlação espacial entre os pontos de medida pode ser quantificada por meio da função de variância (Eq. (4))

$$
y=(x, h) \frac{1}{2} \operatorname{var}[F(x)-F(x+h)]
$$

onde $h$ é a distância euclidiana entre dois pontos e que a tendência é constante, e $y(x, h)$ são independentes de $x$. Uma função paramétrica é usada para modelar a variância para valores diferentes de $h$.

Cada krigagem considerou uma quantidade máxima de 20 referências, com máxima uniformidade na distribuição entorno do ponto alvo, a uma distância máxima de $500 \mathrm{~km}$ do centro do quadrante.

\subsection{A construção do índice}

O Índice de Proporção de Escalas (IPE) é um índice aqui proposto e é baseado em estatísticas de centralização e de dispersão de dados de precipitação, os quais devem ser estruturados no espaço e no tempo. O método de janelas móveis (moving Windows ou Kernels) busca analisar a centralização por média aritmética simples de uma série espacial ou temporal de dados, bem como a sua dispersão, geralmente por meio do desvio padrão. A técnica é comumente utilizada em estudos pluviométricos, geralmente considerando as dimensões espacial e temporal isoladamente. Por exemplo, Zhang et al. (2015) e Pardo-Igúzquiza, et al. (2005) utilizaram janelas móveis espaciais para mapear e avaliar incertezas em valores locais de precipitação. Peters et al. (2014) utilizaram a técnica para separar valores de precipitação de possíveis ruídos em séries temporais e Horton et al. (2017) utilizaram janelas móveis temporais para prever a precipitação em escala sub-diária. Neste artigo, a abordagem de janelas móveis consiste na integração das dimensões espaço e tempo. Assim, pretende-se descrever a variabilidade pluviométrica de um local, por meio de um índice que represente a maior (ou menor) influência do espaço em relação ao tempo, método até então não utilizado em estudos de variabilidade pluviométrica.

O conjunto de dados utilizado na construção do IPE está representado na Fig. 1 e considera um conjunto de da-

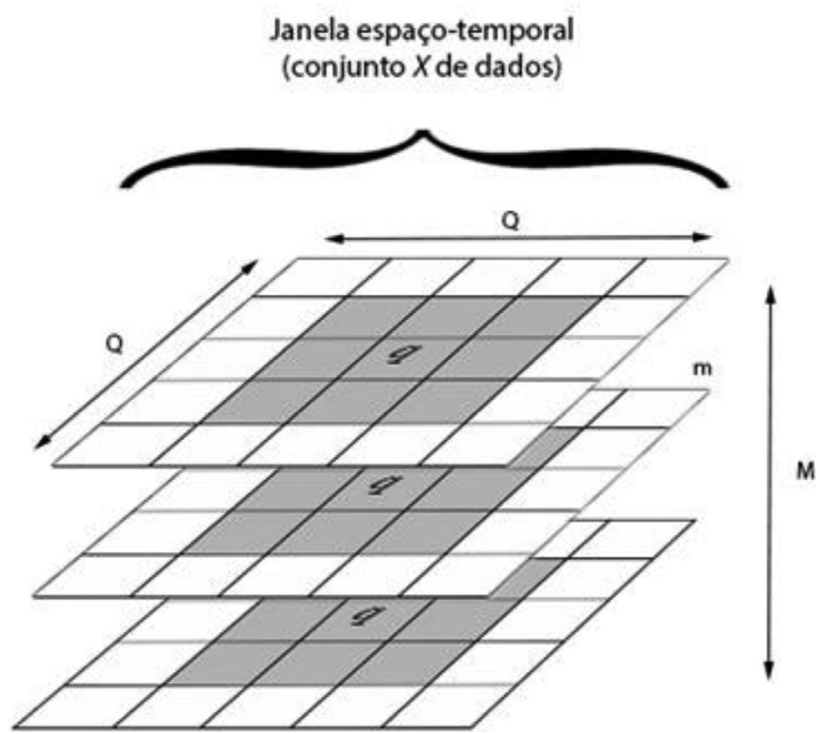

Figura 1 - Esquema ilustrando um conjunto de dados pluviométrico para a construção do Índice de Proporção de Escalas (IPE). Os $X$ valores correspondem a uma janela temporal unidimensional (considerando a linha temporal) e a uma janela espacial bidimensional (considerando as dimensões latitude e longitude). A janela temporal é composta pelos valores imediatamente vizinhos ao mês de interesse e, portanto, composta por 3 meses $\left(M=3^{1}\right)$, enquanto a janela espacial, ao considerar os quadrantes imediatamente vizinhos, é composta por um conjunto de 9 quadrantes $\left(Q=3^{2}\right)$.

dos de precipitação (X) numa escala espaço-temporal de $Q$ quadrantes e $M$ meses. Contudo, o índice é um indicador exclusivo para uma janela cujo centro é representado pelo quadrante $q$ e o mês $m$. Assim, um valor específico de IPE pode ser identificado por $I P E_{(M, Q, m, q)}$, representando uma série espaço-temporal que pode ser analisada segundo suas estatísticas de tendência central e dispersão. Para esse fim, são empregadas janelas móveis, em que o valor central ( $q$ e $m$ ) corresponde a uma média aritmética simples dos valores vizinhos da janela espaço-temporal. Matematicamente o método é caracterizado como um tipo de convolução, um exemplo de filtro passa-baixa usado no processamento de sinais (Skrøvseth et al., 2012) e foi aplicado a janelas de 3 meses (tempo unidimensional) e 3 quadrantes (espaço bidimensional), de modo a obter uma medida mínima de dispersão. Assim, o cálculo do IPE para o mês de fevereiro no quadrante de latitude -3 e longitude -45 considera respectivamente os valores de precipitação dos meses de janeiro e março, das latitudes -2 e -4 e das longitudes -44 e $-46$.

A medida de dispersão mais básica possível, baseada na totalidade dos dados do conjunto $X$ é obtida pela análise dos desvios da média, ou simplesmente desvios. Considerando que, invariavelmente, a média dos desvios corresponde a zero, é fundamental analisar os desvios em termos de seu módulo (ausência de sinal), de forma que desvios negativos e positivos não se anulem e que os resíduos possam ser computados integralmente e linearmente, enquanto expressão da variação dos dados. A Eq. (5) apre- 
senta o cálculo para a obtenção da Média dos Módulos dos Desvios no tempo para a escala de $M x Q$ e, de forma análoga, para análise da dispersão promovida pelo espaço, assume-se a Eq. (6).

$$
D t_{(M, Q)}=\frac{1}{M} \sum_{t=M_{1}}^{M_{M}}\left|\bar{x}_{Q}-x_{t, Q}\right|
$$

onde $D t_{(M, Q)}$ : Média dos módulos dos desvios no tempo para escala de $M$ meses e $Q$ quadrantes; $\bar{x}_{Q}:$ Média de pluviosidade para os $M$ meses e na escala espacial de $Q$ quadrantes; $x_{(t, Q)}$ : pluviosidade para o tempo $t$ e escala espacial $Q ; M$ : escala temporal (número de meses); $Q$ : escala espacial (número de escala); $M_{1}$ : primeiro mês da janela temporal; $M_{M}$ : último mês da janela temporal.

$$
D e_{(M, Q)}=\frac{1}{Q} \sum_{e=Q_{1}}^{Q_{Q}}\left|\bar{x}_{M}-x_{M, e}\right|
$$

onde $D e_{(M, Q)}$ : Média dos módulos dos desvios no espaço para a escala de $M$ meses e $Q$ quadrantes; $\bar{x}_{M}$ : Média de pluviosidade para as $Q$ quadrantes na escala temporal de $M$ meses; $x_{(M, e)}$ : pluviosidade para o mês $M$ e escala espacial $e$ $M$ : escala temporal (número de meses); $Q$ : escala espacial (número de quadrantes); $Q_{1}$ : primeira quadrante da janela espacial; $Q_{Q}$ : última quadrante da janela espacial.

O IPE é obtido pela média do módulo dos desvios no tempo (Dt) para a escala de $M x Q$, dividida pela soma de ambas as médias dos desvios, no tempo e no espaço (De) (Eq. 7).

$$
I P E_{(M, Q)}=\frac{D t_{(M, Q)}}{D t_{(M, Q)}+D e_{(M, Q)}}
$$

onde IPE: Índice de Proporção de Escalas; $0 \leq I P E \geq 1$;

Assim:

- O valor de IPE aproxima-se a 0 quando o De é maior que o $D t$, indicando que a variação na quantidade de chuva deve-se em maior parte à heterogeneidade espacial;

- O valor de $I P E$ aproxima-se a 1 quando o $D t$ é maior que o De, indicando que a variação na quantidade de chuva deve-se em maior parte à heterogeneidade temporal;

- O valor de IPE em torno de 0,5 ocorre quando os Dt e De apresentam parcelas semelhantes de variação, ou seja, quando a quantidade de chuva deve-se de forma semelhante à heterogeneidade espacial e temporal.

\section{Resultados e Discussão}

O IPE é um índice proposto para representar de forma sintética a variabilidade espaço-temporal da precipitação. Sua concepção, explicada tão somente pelas relações algébricas fundamentais da Eq. (7), torna implícita a existência de correlações positivas com a variabilidade temporal (altos valores de $I P E$ ) e negativas com a variabilidade espacial (baixos valores de IPE).
A Tabela 1 mostra a correlação entre estas variáveis, confirmando a aderência estatística entre IPE e $D t$ $(\mathrm{r}=0,50344)$ e entre $I P E$ e $D e(\mathrm{r}=-0,46719)$. A maior correlação entre $I P E$ e $D t$ indica a maior variabilidade na distribuição das chuvas no Brasil em função do tempo, inclusive ocorrendo casos em que, apesar das chuvas apresentarem grandes desvios no espaço, os $D t$ promovem maior dispersão nas chuvas e, portanto maiores IPEs. Um significado climatológico interessante está na correlação entre IPE e PLSD ( $\mathrm{r}=0,069932)$ que, mesmo sendo baixíssima, possui significância estatística confirmada em decorrência do elevado grau de liberdade nos dados não agregados (n $9.708=12$ meses x 809 quadrantes), indicando que os altos IPEs tendem a ocorrer em condições de maior volume pluviométrico. Porém, a melhor compreensão da significância estatística entre $I P E$ e $P L S D$, se dá pelas altas e significativas correlações positivas entre $P L S D$ e $D t$ $(\mathrm{r}=0.53365)$ e $P L S D$ e $D e(\mathrm{r}=0.45129)$, que confirmam o efeito do volume pluviométrico sobre a variabilidade no tempo e no espaço. Sendo estas duas correlações positivas e significativas, tem-se consequentemente correlação positiva entre $D t$ e $D e(\mathrm{r}=0,40929)$, indicando que, até certo ponto, as condições de maiores desvios no tempo correspondem às condições de maiores desvios no espaço e vicee-versa. A fraca correlação entre $I P E$ e $P L S D$ não representa limitações para a utilização do índice, ao contrário. $\mathrm{O}$ IPE representa justamente os resíduos da regressão linear (validada estatisticamente) entre a variabilidade espacial e temporal das chuvas, muito mais que pelo volume em si. Em outras palavras, o IPE é um indicador de falta de aderência e correlação do modelo de regressão, onde existem outliers, os quais são mais importantes que as fortes e significativas correlações.

Quando realizada a mesma análise para os dados agregados espacialmente no Brasil, discriminados apenas pelos 12 meses do ano (n 12) (Tabela 2), as conclusões são análogas. As correlações continuam fortes e significativas, existem fortes associações entre volume pluviométrico e variabilidade espacial e temporal, sendo o IPE o fator com menor correlação com volumes pluviométricos. Também se mantém as fortes correlações entre variabilidade no espaço e no tempo. A única diferença está na fraca e não

Tabela 1 - Coeficientes de Pearson (r) para correlação linear entre Pluviosidade Média Diária $(P L S D)$, Desvios no Tempo $(D t)$, Desvios no Espaço $(D e)$ e Índice de Proporção de Escalas (IPE), em escala anual para os 809 quadrantes (n $9.708=12$ meses x 809 quadrantes).

\begin{tabular}{lcccc}
\hline & $P L S D$ & $D t$ & $D s$ & $I P E$ \\
\hline$P L S D$ & 1.00 & & & \\
$D t$ & $0.53365^{(*)}$ & 1.00 & & \\
$D s$ & $0.45129^{(*)}$ & $0.40929^{(*)}$ & 1.00 & \\
$I P E$ & $0.069932^{(*)}$ & $0.50344^{(*)}$ & $-0.46719^{(*)}$ & 1.00 \\
\hline
\end{tabular}

$\left.{ }^{*}\right) \mathrm{p}<0.05$. 
significativa correlação entre IPE e De $(\mathrm{r}=0,0096692)$, nesse caso explicada pela generalização espacial, em que os 809 quadrantes foram agregados em um único. Novamente, os dados sugerem que o IPE é um indicador de condições (locacionais ou temporais) de falta de aderência (resíduos) de um modelo linear de dependência espaço-temporal, porém sem significativo efeito do volume pluviométrico $(\mathrm{r}=0.13919)$.

A Fig. 2 apresenta os dados de $P L S D, D t, D e$ e $I P E$ discriminados por meses, genericamente para todo o Brasil, permitindo visualizar que a fraca correlação entre $P L S D$ e IPE, destacada anteriormente, se dá pelos altos valores de IPE serem mais frequentes nos meses de transição entre as estações seca e úmida, enquanto que baixos valores ocorrem tanto nos períodos de maior e de menor pluviosidade. Os dados assim analisados não são totalmente adequados para a efetiva compreensão do IPE e suas peculiaridades, mas contribuem para identificar as especificidades no espaço e no ciclo sazonal, como a forte correlação com a variabilidade no tempo $(D t, \mathrm{r}=0,82416$, Tabela 2), se analisados junto às (Figs. $3 \mathrm{a}$ e b). Por exem-

Tabela 2 - Coeficientes de Pearson (r) para correlação linear entre valores médios mensais (n 12) de Pluviosidade Média Diária (PLSD), Desvios no Tempo $(D t)$, Desvios no Espaço $(D e)$ e Índice de Proporção de Escalas (IPE).

\begin{tabular}{lcccc}
\hline & $P L S D$ & $D t$ & $D e$ & $I P E$ \\
\hline$P L S D$ & 1.00 & & & \\
$D t$ & $0.82416^{(*)}$ & 1.00 & & \\
De & $0.87938^{(*)}$ & $0.7833^{(*)}$ & 1.00 & \\
$I P E$ & 0.13919 & $0.59494^{(*)}$ & 0.0096692 & 1.00 \\
\hline
\end{tabular}

$(*) \mathrm{p}<0.05$. plo, nos meses de junho a agosto, os mais secos do ano na maior parte do Brasil, os valores de $D t$ são altos apenas no extremo norte do país (Figs. 3a e b). Na primavera os $D t$ aumentam e adquirem uma configuração zonal sobre o centro do país, que se fortalece e se estabelece a sudeste para, a partir de dezembro, adquirir um sentido meridional até o litoral norte e Roraima. Em março retorna o padrão zonal que migra progressivamente para oeste numa configuração mais meridional até o litoral norte, recolhendo-se novamente para o extremo norte do país a partir de junho. De modo geral, os $D t$ estão positivamente relacionados ao volume pluviométrico, similarmente a estatística de desvio padrão dos dados pluviométricos, onde locais mais úmidos e períodos de transição no regime pluviométrico frequentemente estão associados a desvios padrões elevados.

Apesar do elevado volume pluviométrico da região norte, alguns locais apresentam baixos $D t$, como a porção oeste da Amazônia, que Bernardo e Molion (2004) sugerem apresentar uma baixa variabilidade sazonal da precipitação em função da baixa variabilidade de radiação de ondas longas, responsável pela manutenção da cobertura de nuvens e movimentos convectivos constantes ao longo do ano.

Os baixos $D t$ na região sul do Brasil, provavelmente estão relacionados à baixa variabilidade intra-anual do regime pluviométrico, em virtude das sucessivas invasões da Frente Polar Atlântica ao longo do ano, cuja pluviosidade associada, possui caráter menos intermitente e de ampla abrangência espacial (Nimer, 1979; Grimm et al., 2007).

Enquanto o De reflete maior heterogeneidade pluviométrica em menores distâncias, o $D t$ reflete maior hetero-

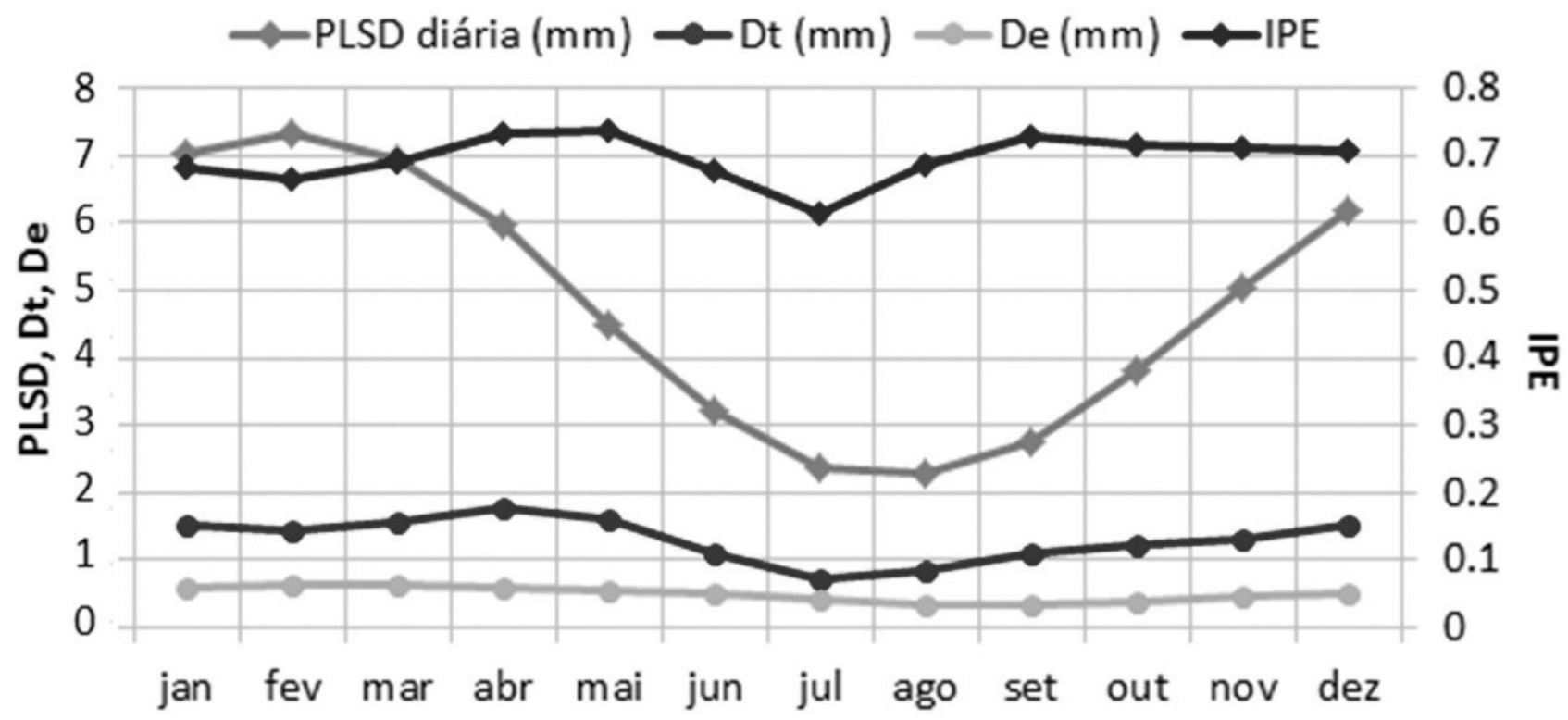

Figura 2 - Representação espaço-temporal dos valores de Pluviosidade Média Diária (PLSD), Desvios no Tempo (Dt), Desvios no Espaço (De) e Índice de Proporção de Escalas (IPE) para a série pluviométrica mensal de 1975 a 2010, no Brasil. 

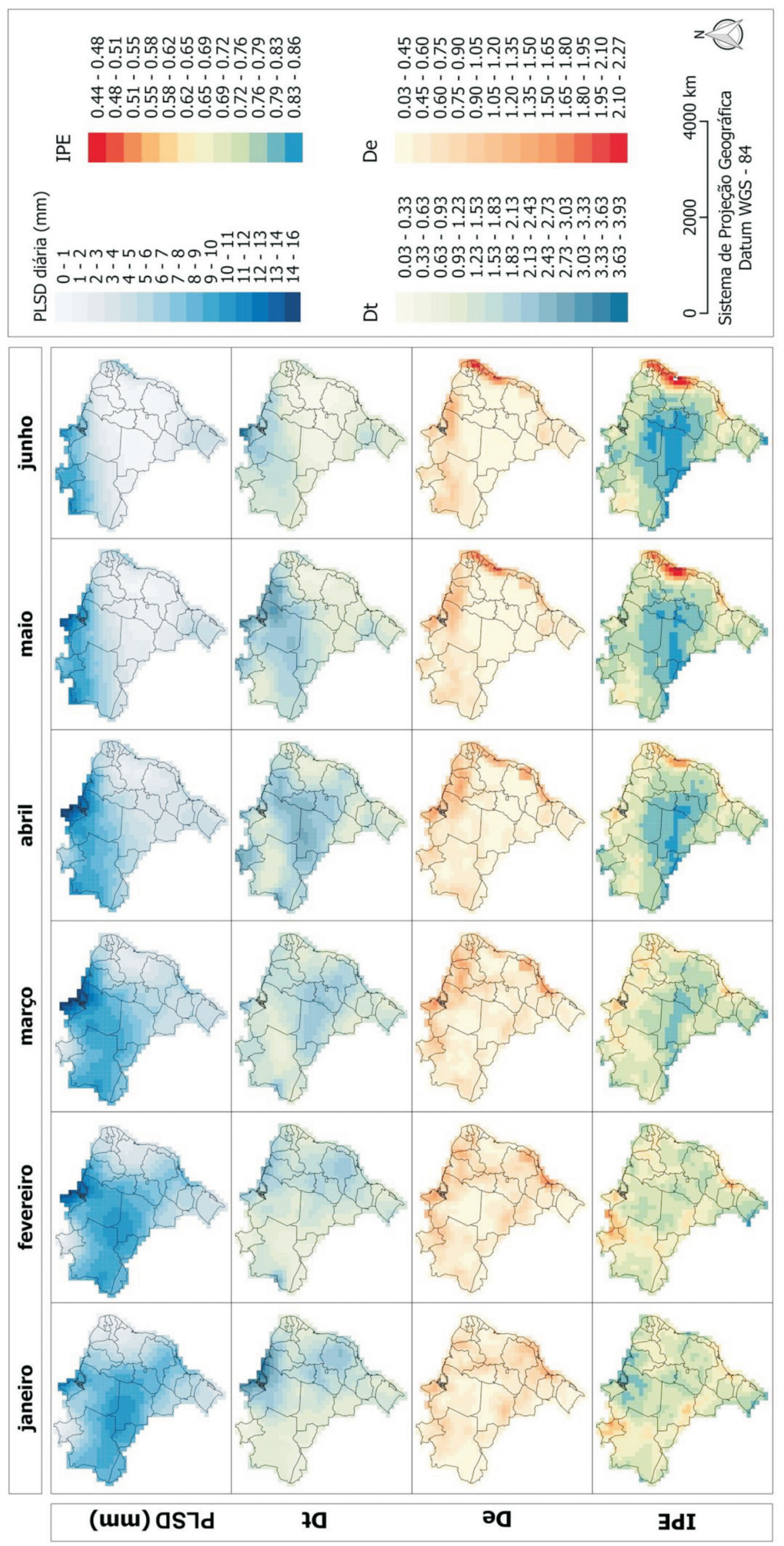

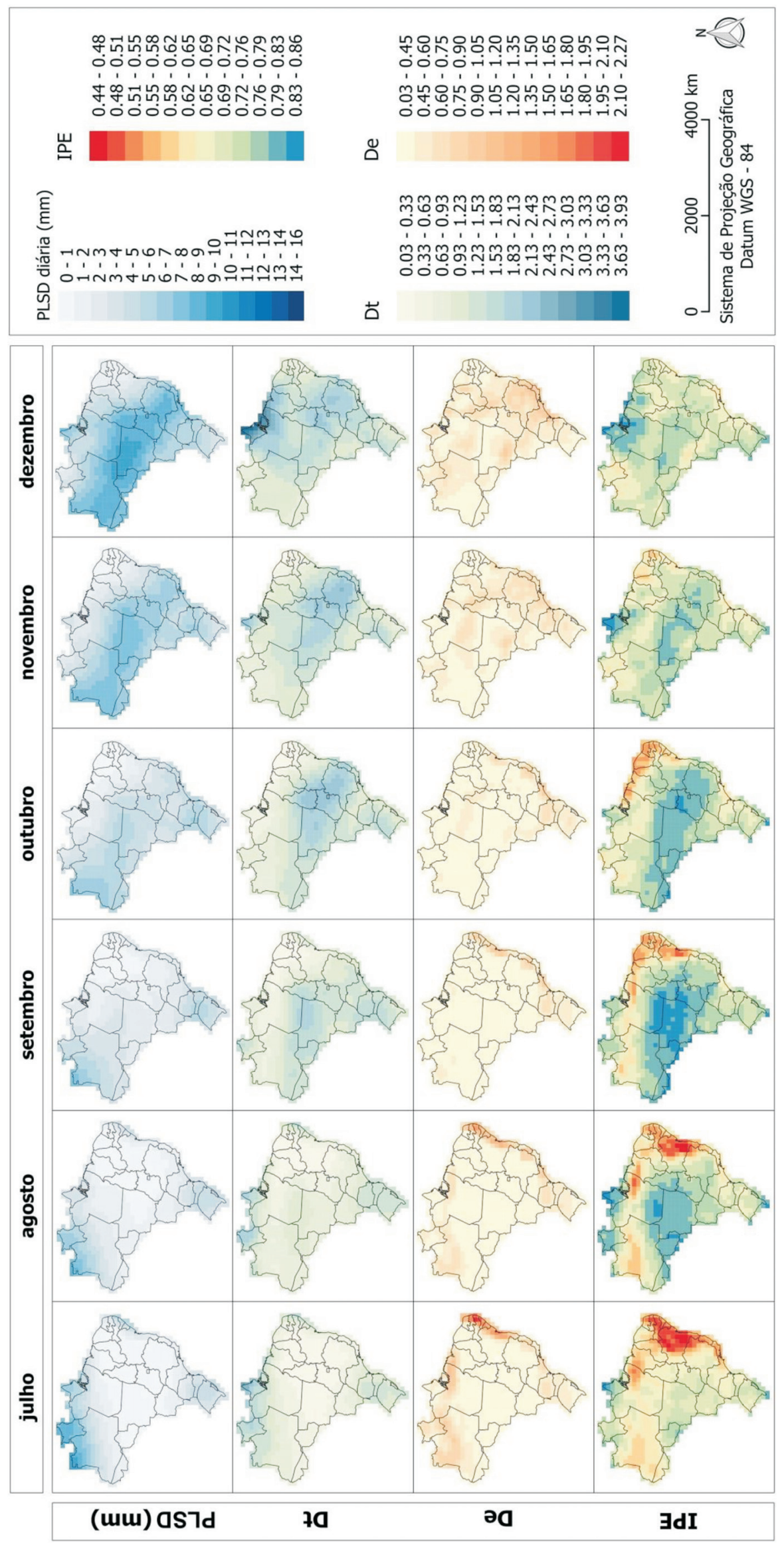
geneidade pluviométrica em curtos períodos de tempo (Figs. 3a e b), o que é atribuído à propriedade de escala a que são submetidos os fenômenos geográficos.

$\mathrm{Na}$ escala anual (Fig. 4), os maiores valores de IPE (entre 0,76 e 0.84 ) ocorrem na porção central do Brasil, cobrindo quase a totalidade de estado do Mato Grosso, Goiás e parte dos estados vizinhos (Tocantins, Rondônia, Pará, Amazonas e Minas Gerais). Outro local de valores altos de IPE está situado no extremo sul do Rio Grande do Sul, atingindo o valor recorde na média anual $(0,93)$. A partir destes locais, o IPE decresce concentricamente, com valores menores $(\sim 0,60)$ no extremo norte do país (Roraima, norte do Amazonas e nordeste do Pará) e por toda a Costa Atlântica, até o norte do Rio Grande do Sul. Nos estados de São Paulo, Paraná e Santa Catarina, os mais baixos valores de IPE adentram o continente pela costa leste em cerca de $400 \mathrm{~km}$, com valores entre 0,59 a 0.67 , enquanto que, nos estados nordestinos, marcadamente na Bahia, em Alagoas e Pernambuco, ocorrem os menores valores do país $(0,41$ a 0,50$)$ alcançando distâncias em torno de $800 \mathrm{~km}$ da costa.

\subsection{Identificação de padrões espaciais de IPE}

Dada a notável variação sazonal e geográfica dos padrões de $I P E$, faz-se necessária uma avaliação mais detalhada e que permite identificar, pelo menos, cinco padrões regionais principais, que podem ser descritos paralelamente aos principais sistemas meteorológicos que definem o regime pluviométrico e as peculiaridades locais do relevo (Fig. 4).

Padrão A: O Padrão A descreve uma faixa de baixos IPEs do litoral norte gaúcho ao litoral leste do estado do Rio Grande do Norte. De abril a julho, há uma intensificação da influência do espaço na variabilidade pluviométrica, seguindo de uma retração contínua do litoral sudeste ao litoral norte até novembro, onde os baixos IPEs permanecem por mais dois meses. No restante do período (dezembro a março), esse padrão permanece na costa brasileira, porém, de forma menos evidente.

O Padrão A corresponde à região mais estudada em termos de interação entre relevo e precipitação no Brasil, devido as suas peculiaridades de altitude, direção de vertentes, posição e configuração espacial, que lhe conferem um caráter complexo quando comparado ao restante do país. a)

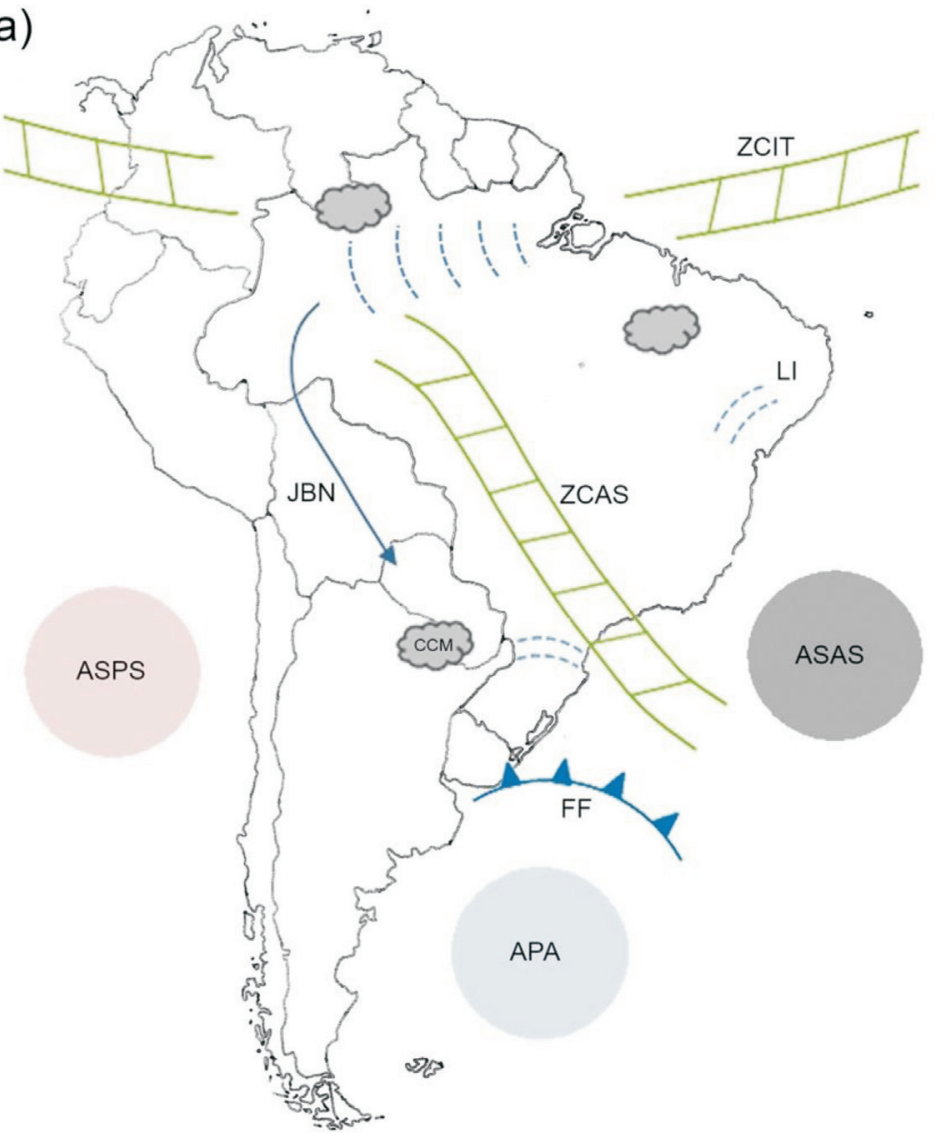

b)

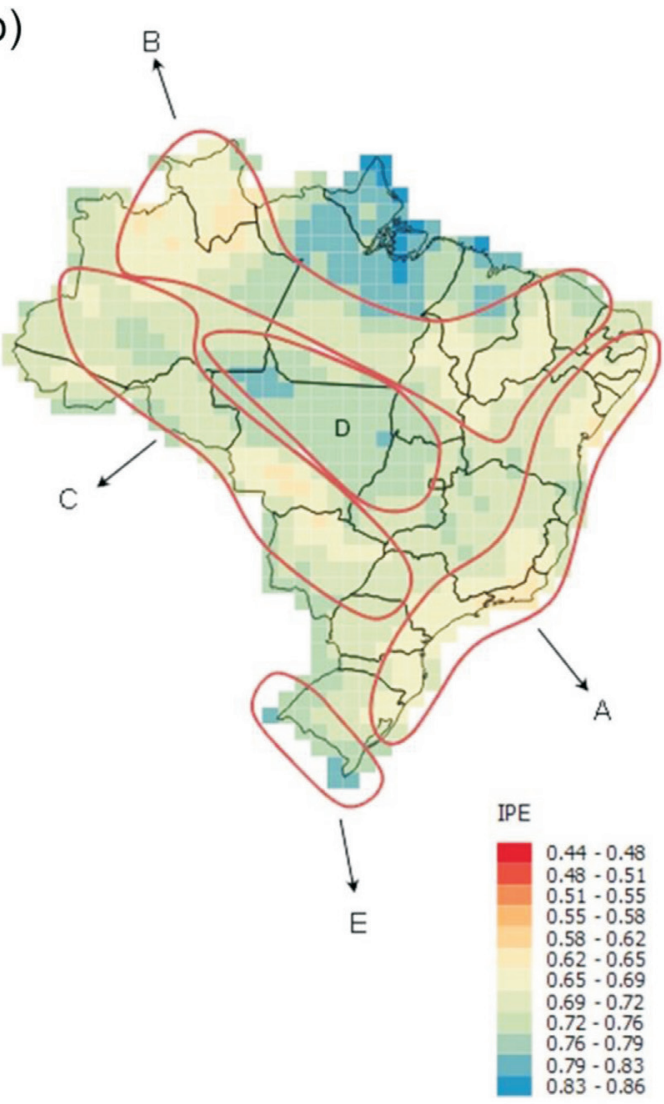

Figura 4 - Principais sistemas que atuam sobre o tempo meteorológico (a) e que promovem a variabilidade espaço-temporal das chuvas no Brasil (b), definindo os padrões (de A até E) identificados com base no Índice de Proporção de Escalas. ASPS: Anticiclone Semipermanente do Pacífico Sul; ASAS: Anticiclone Semipermanente do Atlântico Sul; ZCIT: Zona de Convergência Intertropical; ZCAS: Zona de Convergência do Atlântico Sul; CCM: Complexos Convectivos de Mesoescala; JBN: Jatos de Baixos Níveis; FF: Frente Fria; LI: Linhas de Instabilidade; APA: Anticiclone Polar Atlântico. 
Dentre os trabalhos que confirmam essa interação estão Forgiarini e Vendruscolo (2013); Baratto e Wollmann (2015), no Rio Grande do Sul, Rodrigues (2015) em Santa Catarina, Correa (2013) no Paraná, Milanesi e Galvani (2011); Soares et al. (2014), no Rio de Janeiro, Seluchi et al. (2011) na Serra do Mar, Ávila et al. (2009) na Serra da Canastra, Lyra et al. (2014). Pereira (2013) em Alagoas, Souza et al. (2015) em Pernambuco, Schmidt (2014) no Rio Grande do Norte.

Sobre o litoral do Paraná e São Paulo, ao longo da Serra do Mar, ocorrem valores de IPE relativamente baixos $\mathrm{o}$ ano todo, principalmente de janeiro a abril. Nesse período os principais mecanismos de precipitação são Zona de Convergência de Atlântico Sul (ZCAS), além das frequentes passagens de Frentes Frias (FF), que tanto interagem com a ZCAS, como com as elevações do litoral. Além disso, o efeito topográfico é intensificado pelos ventos de sudeste, do Anticiclone Subtropical do Atlântico Sul (ASAS) que sopram alinhados à topografia da Serra do Mar (Grimm e Zilli, 2009).

O litoral sudeste apresenta alta sazonalidade pluviométrica devido à atuação e interação de inúmeros sistemas tropicais e intertropicais, responsáveis por uma estação chuvosa de verão que constrasta com os baixos volumes da estação seca, em decorrência da subsidência gerada pela proximidade do ASAS, no inverno. No verão é frequente a ocorrência da ZCAS, com sentido noroeste-sudeste sobre a região, além da frequente permanência das $\mathrm{FF}$, em torno da latitude $22^{\circ} \mathrm{S}$, devido ao equilíbrio dinâmico entre o Anticiclone Migratório Polar e o ASAS, cuja estacionaridade do fluxo atmosférico interage com o relevo e influencia a ocorrência de chuvas (Grimm et al., 2007; Nunes et al., 2009). A elevada heterogeneidade temporal das chuvas durante o verão mantém os IPEs altos nesse período, apesar de haver uma forte heterogeneidade espacial próxima a costa, nas maiores elevações. A redução da heterogeneidade temporal nos meses mais secos, permite evidenciar o efeito da topografia sobre os baixos valores de IPE.

$\mathrm{Na}$ costa leste do nordeste brasileiro, o máximo volume pluviométrico ocorre de maio a julho. Dentre os principais sistemas relacionados está à circulação de brisa mais intensa que advecta bandas de nebulosidade para o continente, a ação das FF remanescentes que se propagam ao longo da costa (Kousky, 1979) e perturbações ondulatórias no campo dos alísios, associados aos sistemas frontais (SF), com os ventos de leste (Chan, 1990; Gomes, 2012). Além disso, de abril a agosto, quando o ASAS está mais próximo à região, se estabelecem ventos de sudeste, perpendiculares a costa (Servain e Lukas, 1990). Na transição para a estação chuvosa e ao longo dessa, a heterogeneidade temporal aumenta e os ventos passam a soprar do quadrante leste, alinhados à topografia, diminuindo a heterogeneidade espacial das chuvas. É, portanto o período em que os IPEs são mais altos em toda a região nordeste.
Padrão B: O Padrão B ocorre do litoral norte do Rio Grande do Norte ao oeste da região amazônica, onde os baixos IPEs são mais evidentes, formando uma faixa contínua, de julho a agosto e, secundariamente de fevereiro a abril. No primeiro caso, o baixo volume pluviométrico, associado à ausência da Zona de Convergência Intertropical (ZCIT) (Kousky, 1979; Carvalho e Oyama, 2013), reduz a heterogeneidade temporal das chuvas a qual é acompanhada de baixos valores de IPEs, que aumentam quando a ZCIT inicia seu deslocamento para o sul, sobre a região. Observa-se uma relação mais clara entre o IPE e a ZCIT nos meses de fevereiro e março, quando ocorre o chamado "salto", caracterizado como um retorno de aproximadamente $1,5^{\circ}$ da ZCIT para o norte (Uvo e Nobre, 1989; Carvalho e Oyama, 2013) e que é acompanhado da redução do IPE. Na sequência, quando o deslocamento da ZCIT para o sul é retomando, os valores de IPE se elevam.

No final da primavera austral, a ZCIT inicia seu deslocamento para o litoral norte (Carvalho e Oyama, 2013) e os alísios de nordeste passam a predominar sobre o estado de Roraima, com altas velocidades devido ao progressivo aquecimento do continente. Esses sofrem aceleração por efeito de compressão vertical nos Planaltos do Norte da Amazônia, na porção norte do estado e noroeste do Pará (Amarante, Zack e Sá, 2001). Provavelmente este processo é responsável pela redução do IPE.

As chuvas no oeste da Amazônia são bem distribuídas no ano, devido a atuação contínua da massa equatorial continental (Marengo, e Hasternrath, 1993; Santos et al., 2015) que mantém uma baixa heterogeneidade temporal das chuvas, permitindo a expressão dos baixos valores de IPE. No inverno austral, devido a redução do fluxo sobre os trópicos, apenas os ventos da porção sudeste amazônica são canalizados como Jatos de Baixos Níveis (JBN), para as altas latitudes e, portanto, a maior parte dos ventos amazônicos seguem preferencialmente para o leste dos Andes, pela depressão do rio Amazonas, e são elevados pela topografia (Da Rocha et al., 2009). É difícil identificar o que eleva o efeito do espaço nos meses de inverno, porém, Betts et al. (2009) salienta que apesar da topografia local não ser muito pronunciada, pequenas diminuições da forçante temporal fazem com que a condição local afete bastante a distribuição das chuvas.

Padrão C: A partir de dezembro surge um terceiro padrão de forte efeito do espaço, que se estende da Amazônia central, na direção sudoeste, pelo vale do Rio Madeira até a tríplice fronteira Rondônia-Amazonas-Bolívia, defletindo para o sudeste, sobre a Bacia do Prata até atingir o litoral paulista. Esse padrão é melhor observado nos meses em que a ZCAS é mais ativa, se estabelecendo nas adjacências da região. Nesse período, o fluxo sobre os trópicos se intensifica e os jatos de baixos níveis, ao escoarem para o sul fornecem umidade, ora para a região da ZCAS, ora para os Complexos Convectivos de Mesoescala 
(CCM) que se originam sobre o norte da Argentina (Marengo, 2005).

Sistemas frontais do sul do país também contribuem para a precipitação nessa região (Amorim Neto et al., 2015). Em janeiro e fevereiro esse escoamento parece gerar elevada heterogeneidade espacial e baixos IPEs sobre a margem mais larga do Rio Madeira, ao sul do Amazonas e, de novembro a março, sobre o sul do Mato Grosso e norte do Mato Grosso do Sul, devido a elevada heterogeneidade espacial no relevo do planalto e Chapada dos Parecis e a Serra do Rio Guaporé. No inverno, a localização dos JBN mais ao sul (Poveda et al., 2014) sugerem uma explicação para os baixos IPEs, que nesta época ali ocorrem.

Padrão D: Os padrões descritos anteriormente articulam-se, formando um arco que se fecha num grande círculo, composto por baixos valores de IPE. No interior desse círculo distingue-se o padrão $\mathrm{D}$ que consiste em altos valores de IPE durante todo o ano, porém, ligeiramente mais elevados na primavera e outono. A região corresponde à área de atuação da ZCAS, considerada um dos principais sistemas determinantes do regime de chuvas dessas regiões (Barreiro e Chang, 2002; Grimm, 2011; Ambrizzi e Ferraz, 2015), cuja atividade convectiva pode ser intensificada pelo fluxo de umidade transportado pelos jatos de baixos níveis ao escoarem para o sul do continente. Nos meses de inverno, o bloqueio gerado pela alta pressão do ASAS, que se estende sobre o país, inibe a chegada dos sistemas geradores de chuva e a região permanece sob a atuação da massa tropical continental, quente e seca (Vianello e Alves, 1991; Lübbecke et al., 2014). Estes sistemas meteorológicos (SF, ASAS, ZCAS) que parecem reger o Padrão D são altamente variáveis no tempo, porém notoriamente constantes no espaço, visto apresentarem continuidade em extensões de centenas ou milhares de quilômetros e, portanto, são típicos mecanismos promotores de altos valores de IPE.

Padrão E: O último padrão identificado (E) é representado por uma pequena área no extremo sul do Brasil, com altos IPEs, os quais sofrem uma leve redução nos meses de inverno e primavera. A homogeneidade espaçotemporal das chuvas parece ter relação com os baixos contrastes do relevo típicos do extremo sul do Rio Grande do Sul e com a frequente passagem de FF (Siqueira e Machado, 2004; Pampuch e Ambrizzi, 2016) que mantém os IPEs elevados. Também ocorrem altos valores de IPE no oeste da região durante a primavera, verão e outono, respectivamente, quando é mais frequente a ocorrência de CCM que se originam sobre o Paraguai e Argentina, se desenvolvem e migram para a bacia do Prata. Desta forma, representa um mecanismo análogo àquele que explica o Padrão D.

\section{Conclusão}

Por ser um índice espacialmente explícito e organizado regularmente no espaço geográfico, o IPE mostra-se próspero para estudos relacionados a variabilidade espaçotemporal das chuvas, já que, ao considerar todo o território brasileiro, reduz a subjetividade na comparação entre entidades geográficas distintas.

O IPE permitiu identificar cinco padrões representativos da variabilidade espaço-temporal das chuvas no Brasil e apresenta-se promissor enquanto um indicativo da participação dos diferentes sistemas de tempo meteorológico, desde aqueles de âmbito local até os tipicamente sinóticos.

O IPE foi aqui expresso por um único número para cada quadrante na resolução anual, ou doze números para resolução mensal. Assim, não nos preocupamos em identificar tendências temporais do IPE para a série temporal, ou mesmo identificar rupturas, à exemplo do que fizeram Villar et al. (2009) e Fetter et al. (2016) para séries pluviométricas. Obviamente, tais análises são factíveis mediante algumas adaptações nos procedimentos computacionais ora apresentados, sendo razoável supor que processos históricos, como o desmatamento, a conurbação ou mesmo o alagamento provocado por grandes reservatórios e complexos hidrelétricos, possam mostrar tendências ou rupturas nos valores de IPE. Neste sentido, é fundamental que se avalie a compatibilidade do processo gerador de uma suposta mudança diante das resoluções espacial e temporal das chuvas. Essa é uma questão que poderia ser investigada, por exemplo, pela aplicação do IPE a distintas séries temporais (janelas temporais), como em escala de dias ou horas. Isso possibilitaria distinguir o efeito dos fatores estáticos, como o relevo, frente aos dinâmicos, como as mudanças de uso da terra ou o aquecimento da baixa troposfera observado nas últimas décadas, ou mesmo, a importância de sistemas de tempo efêmeros, responsáveis por catástrofes e danos na esfera socioeconômica e ecológica. Trata-se de adequar o grupo de dados em investigações futuras, visando melhor analisar o fenômeno de interesse. Iniciativas desta alçada ajudariam a complementar a avaliação do IPE. Por outro lado, estabeleceria um desafio de esforço computacional consideravelmente maior.

\section{Referências}

AMARANTE, O.A. C.; ZACK, M.B.E.J.; SÁ, A.L. Atlas do Potencial Eólico Brasileiro. CRESESB - Centro de Referência para Energia Solar e Eólica Sérgio Brito, p. 45, 2001.

AMBRIZZI, T.; FERRAZ, S.E.T. An objective criterion for determining the South Atlantic Convergence Zone. Frontiers in Environmental Science, v. 3, p. 9, 2015.

AMORIM NETO, A.D.C.; SATYAMURTY, P.; CORREIA, F.W. Some observed characteristics of frontal systems in the Amazon Basin. Meteorological Applications, v. 22, n. 3, p. $617-635,2015$.

ÁVILA, L.F.; DE MELLO, C.R.; VIOLA, M.R. Mapeamento da precipitação mpinima provável para o sul de Minas Gerais. Revista Brasileira de Engenharia Agrícola e Ambiental, v. 13, p. 906-915, 2009. 
BARATTO, J.; WOLLMANN, C.A. Perfil topo-oro-pluviométrico do estado do Rio Grande do Sul, Brasil. Interface, v. 10, p. 40-55, 2015.

BARGAOUI, Z.K.; CHEBBI, A. Comparison of two kriging interpolation methods applied to spatiotemporal rainfall. Journal of Hydrology, v. 365, n. 1-2, p. 56-73, 2009.

BARREIRO, M.; CHANG, P. Variability of the South Atlantic Convergence Zone simulated by an Atmospheric General Circulation Model. Journal of Climate, v. 15, p. 745-763, 2002.

BARROS, A.P.; LETTENMAIER, D.P. Dynamic modeling of orographically induced precipitation. Reviews of Geophysics, v. 32, n. 3, p. 265-284, 1994.

BERNARDO, S.O.; MOLION, L.C.B. Variabilidade espacial e temporal da precipitação sobre a América do Sul Tropical e costa oeste do continente Africano Anais XIII, Congresso Brasileiro de Meteorologia, Fortaleza (CE), 2004.

BETTS, A.K.; FISCH, G.; VON RANDOW, C.; SILVA DIAS, M.A.F.; COHEN, J.C.P.; DA SILVA, R.; FITZJARRALD, D.R. The Amazonian boundary layer and mesoscale circulations. In: MIKELLER, M.B.M.; GASH, J.; SILVA DIAS, P. (Ed.). Amazonia and Global Change, v.186, 2009. p.163181. ISBN 0065-8448.

BUSUIOC, A.; CHEN, D.; HELLSTROM, C. Temporal and spatial variability of precipitation in Sweden and its link with the large-scale atmospheric circulation. Tellus A, v. 53, n. 3 , p. 348-367, 2001.

CAMARGO, E.C.G. Geoestatística: fundamentos e aplicações. In: GILBERTO CAMARA e MEDEIROS, J.S.D. (Ed.). Geoprocessamento para Projetos Ambientais. Inpe: Inpe, v.1, 1998. p.36.

CAREY, L. D.; CIFELLI, R.; PETERSEN, W.A.; RUTLEDGE, S.A.; SILVA DIAS, M.A.F. Characteristics of Amazonian rain measured during TRMM-LBA. 30th Conference on Radar Meteorology, v. 12A.9, 2001.

CARVALHO, M.A.V.; OYAMA, M.D. Variabilidade da largura e intensidade da Zona de Convergência Intertropical Atlântica: Aspectos observacionais. Revista Brasileira de Meteorologia, v. 28, n. 3, p. $305-316,2013$.

CHAN, S.C. Analysis of easterly wave disturbances over South Atlantic Ocean. Dissertação de mestrado, Instituto Nacional de Pesquisas Espaciais - INPE - 5222 - TDL/437, p. 104, 1990.

CORREA, M.G.G. Distribuição espacial e variabilidade da precipitação pluviométrica na bacia do rio Piquiri-PR. Mestrado em Geografia Física, Universidade de São Paulo, 2013.

DA ROCHA, R.P.; MORALES, C.A.; CUADRA, S.V.; AMBRIZZI, T. Precipitation diurnal cycle and summer climatology assessment over South America: An evaluation of Regional Climate Model version 3 simulations. Journal of Geophysical Research, v. 114, 2009.

FETTER, R.; HENKE-OLIVEIRA, C.; DEBORTOLI, N.; SAITO, C.H. A contribuição da análise espaço-temporal de dados climáticos. In: BURSZTYN, M. E. R. F., S. (Ed.). O clima em transe: Vulnerabilidade e adaptação da agricultura familiar. 1. Rio de Janeiro: Garamond, 2016. cap. 10, p.219-244.

FORGIARINI, F.R.; VENDRUSCOLO, D.S. Análise de chuvas orográficas no centro do estado do Rio Grande do Sul. Revista Brasileira de Climatologia, v. 13, p. 107-119, 2013.
GOMES-FILHO, M.F. Um estudo sobre a influência do albedo diferencial e da orografia na circulação atmosférica: Uma aplicação para o Nordeste Brasileiro. INPE-1640TDL/015, INPE, São José dos Campos (SP), 1979.

GOMES, H. B. Distúrbios Ondulatorios de Leste no Nordeste Brasileiro: climatologia e modelagem numérica. Tese de doutorado em Ciências., p. 99, 2012.

GRIMM, A.M. Interannual climate variability in South America: impacts on seasonal precipitation, extreme events and possible effects of climate change. Stochastic Environmental Research and Risk Assessment, v. 25, n. 4, p. 537-554, 2011.

GRIMM, A.M.; PAL, J.S.; GIORGI, F. Connection between Spring Conditions and Peak Summer Monsoon Rainfall in South America: Role of Soil Moisture, Surface Temperature, and Topography in Eastern Brazil. Journal of Climate, v. 20, n. 24, p. 5929-5945, 2007.

GRIMM, A.M.; ZILLI, M.T. Interannual variability and seasonal evolution of summer monsoon rainfall in South America. Journal of Climate, v. 22, n. 9, p. 2257-2275, 2009.

HARRIS, D.; GEORGIOU, E. F. Multiscale Statistical Properties of a High: Resolution Precipitation Forecast. Journal of Hidrometeorology, v. 2, p. 406-418, 2001.

HORTON, P.; JABOYEDOFF, M.; OBLED, C. Introducing a moving time window in the analogue method for precipitation prediction to find better analogue situations at a sub-daily time step. European Geosciences Union General Assembly 2017. Vienna. 192017.

HOUNSOU-GBO, G.A.; ARAUJO, M.; BOURLÈS, B.; VELEDA, D.; SERVAIN, J. Tropical Atlantic Contributions to Strong Rainfall Variability Along the Northeast Brazilian Coast. Advances in Meteorology, v. 2015, p. 1-13, 2015.

ISAAKS, E.H.; SRIVASTAVA, R.M. An introduction to applied geostatistics. New York: Oxford University Press, 1989.

JAVARI, M. Trend and Homogeneity Analysis of Precipitation in Iran. Climate, v. 4, n. 3, p. 44, 2016.

KAYANO, M.T.; ANDREOLI, R.V. Clima da Região Nordeste do Brasil. In: CAVALCANTI, I.F.A.F.; SILVA DIAS, M.A.F.; JUSTI DA SILVA, M.G.A. (Ed.). Tempo e Clima no Brasil. São Paulo: Oficina de Textos, 2009. cap. 14, p.213-233.

KOUSKY, V.E. Frontal influences on northeast Brazil. Monthly Weather Review, v. 107, p. 1142-1153, 1979.

KOUTSOYIANNIS, D. Nonstationarity vs. Scaling in hydrology. Journal of Hydrology, v. 324, p. 239-254, 2006.

KRIGE, D.G. A statistical approach to some basic mine valuation problems on the Witwatersrand. Journal of the Chemical, Metallurgical and Mining Society of South Africa, v. 52, n. 6, p. 119-139, 1951.

LANDIM, P. M. B. Sobre Geoestatística e mapas. Terra e Didática, v. 2, n. 1, p. 19-33, 2006.

LOUREIRO, G.E.; FERNANDES, L.L.; ISHIHARA, J.H. Spatial and temporal variability of rainfall in the TocantinsAraguaia hydrographic region. Acta Scientiarum, v. 37, n. 1, p. 89-98, 2015.

LÜBBECKE, J.F.; BURLS, N.J.; REASON, C.J.C.; MCPHADEN, M.J. Variability in the South Atlantic Anticyclone and the Atlantic Niño Mode*. Journal of Climate, v. 27, n. 21, p. 8135-8150, 2014.

LYRA, G.B.; OLIVEIRA-JÚNIOR, J.F.; ZERI, M. Cluster analysis applied to the spatial and temporal variability of monthly 
rainfall in Alagoas state, Northeast of Brazil. International Journal of Climatology, v. 34, n. 13, p. 3546-3558, 2014.

MACHADO, L.A.T. Diurnal march of the convection observed during TRMM-WETAMC/LBA. Journal of Geophysical Research, v. 107, n. D20, 2002.

MARENGO, J.; HASTERNRATH, S. Cases studies climatic events in Amazon Basin. Journal of Climate, v. 6, p. 617627, 1993.

MARENGO, J.A. The characteristics and variability of the atmospheric water balance in the Amazon basin: Spatial and temporal variability. Climate Dynamics, v. 24, p. 11-22, 2005.

MILANESI, M.A.; GALVANI, E. Efeito orográfico na Ilha de São Sebastião (Ilhabela - SP). Revista Brasileira de Climatologia, v. 9, p. 68-79, 2011.

NIMER, E. Climatologia do Brasil. Rio de Janeiro: IBGE, p. $422,1979$.

NUNES, L.H.; VICENTE, A.K.; CANDIDO, D.H. Clima da região sudeste do Brasil. In: CAVALCANTI, I.F.A.F.; JUSTI DA, M.G.A.; SILVA DIAS, M.A.F. (Ed.). Tempo e clima no Brasil. São Paulo: Oficina de textos, v.cap 16, 2009. p.243-256.

PAMPUCH, L.A.; AMBRIZZI, T. Sistemas Frontais sobre a América do Sul, Parte II: Monitoramento mensal em dados da reanálise do Ncep/Ncar. Ciência e Natura, v. 38, p. 105, 2016.

PARDO-IGÚZQUIZA, E.; DOWD, P.A.; GRIMES, D.I.F. An automatic moving window approach for mapping meteorological data. International Journal of Climatology, v. 25, p. 665-678, 2005.

PEREIRA, T. Influência do relevo na precipitação das regiões hidrográficas do litoral norte de Alagoas. Espaço e Tempo, v. 33, p. 239-253, 2013.

PETERS, A.; NEHLS, T.; SCHONSKY, H.; WESSOLEK, G. Separating precipitation and evapotranspiration from noise a new filter routine for high-resolution lysimeter data. Hydrology and Earth System Sciences, v. 18, p. 1189-1198, 2014.

POVEDA, G.; JARAMILLO, L.; VALLEJO, L.F. Seasonal precipitation patterns along pathways of South American lowlevel jets and aerial rivers. Water Resources Research, v. 50, n. 1, p. 98-118, 2014.

REINSTORF, F.; BINDER, M.; SCHIRMER, M.; GRIMMSTRELE, J.; WALTHER, W. Comparative assessment of regionalization methods of monitored atmospheric deposition loads. Atmospheric Environment, v. 39, p. 36613674, 2005.

RODRIGUES, M.L.G. Eventos de chuva orografica em Santa Catarina - climatologia e simulações numéricas. Dissertação/Tese em Ciências, Universidade de São Paulo, 2015.

RYSMAN, J.F.; VERRIER, S.; LEMAÎTRE, Y.; MOREAU, E. Space-time variability of the rainfall over the western Mediterranean region: A statistical analysis. Journal of Geophysical Research: Atmospheres, v. 118, n. 15, p. 84488459, 2013.

SANTOS, E.B.; LUCIO, P.S.; SILVA, C.M.S.E. Precipitation regionalization of the Brazilian Amazon. Atmospheric Science Letters, v. 16, n. 3, p. 185-192, 2015.
SCHMIDT, D.M. Dinâmica das configurações de formação e inibição das chuvas no Rio Grande do Norte: caracterização hidrográfica do estado. Tese de doutorado em Ciências Climáticas, p. 136, 2014.

SELUCHI, M.E.; CHOU, S.C.; GRAMANI, M.A case study of a winter heavy rainfall event over the Serra do Mar in Brazil. Geofísica Internacional, v. 50, n. 1, p. 41-56, 2011.

SERVAIN, J.; LUKAS, S. Climatic Atlas of the Tropical Wind Stress and Sea Surface Temperature 1985-1989. Service de la Documentation et des Publications (S.D.P.), Institut Français de Recherch pour l'Exploitation de la Mer, p. 143, 1990.

SILVA DIAS, M.A.F.; DIAS, J.; CARVALHO, L.M.V.; FREITAS, E.D.; SILVA DIAS, P.L. Changes in extreme daily rainfall for São Paulo, Brazil. Climatic Change, v. 116, n. 3-4, p. 705-722, 2012.

SIQUEIRA, J.; MACHADO, L.A.T. Influence of the frontal systems on the day-to-day convection variability over South America. Journal of Climate, 2004.

SKRØVSETH, S.; BELLIKA, J.G.; GODTLIEBSEN, F. Causality in Scale Space as an Approach to Change Detection. PLoS ONE, v. 7, n. 12, 2012.

SOARES, F.S.; FRANCISCO, C.N.; SENNA, M.C.A. Distribuição espaço-temporal da precipitação da região hidrográfica da Baía da Ilha Grande - RJ. Revista Brasileira de Meteorologia, v. 29, n. 1, p. 125-138, 2014.

SOUZA, J.O.P.; ALMEIDA, J.D.M.; CORREA, A.C.B. Caracterização e espacialização da precipitação em bacia hidrográfica com relevo complexo - sertão central Pernambucano - Bacia do Riacho do Saco. Revista de Geografia, v. 32, n. 2, p. 106-126, 2015.

TOKAY, A.; ROCHE, R.J.; BASHOR, P.G. An Experimental Study of Spatial Variability of Rainfall. Journal of Hydrometeorology, v. 15, n. 2, p. 801-812, 2014.

UVO, C.R.B.; NOBRE, C. A zona de convergência intertropical (ZCIT) e a precipitação no Norte do Nordeste do Brasil. Parte I: a posição da ZCIT no Atlântico Equatorial. Climanálise: Boletim de Monitoramento e Análise Climática, v. 4, n. 7, p. 34-40, 1989.

VEMADO, F.; PEREIRA FILHO, A.J. Severe Weather Caused by Heat Island and Sea Breeze Effects in the Metropolitan Area of São Paulo, Brazil. Advances in Meteorology, v. 2016, p. 1-13, 2016.

VIANELLO, R.L.; ALVES, A.R. Meteorologia básica e aplicações. Viçosa. Imprensa Universitária., p. 449, 1991.

VILLAR, E.J.C.; RONCHAIL, J.; GUYOT, J.L.; COCHONNEAU, G.; NAZIANO, F.; LAVADO, W.; DE OLIVEIRA, E.; POMBOSA, R.; VAUCHEL, P. Spatio-temporal rainfall variability in the Amazon basin countries (Brazil, Peru, Bolivia, Colombia, and Ecuador). International Journal of Climatology, v. 29, n. 11, p. 1574-1594, 2009.

ZHANG, G.; YANG, L.; QU, M. Assessing the local uncertainty of precipitation by using moving window geostatistical models. Ecological Informatics, v. 30, p. 133-141, 2015.

This is an Open Access article distributed under the terms of the Creative Commons Attribution Non-Commercial License which permits unrestricted non-commercial use, distribution, and reproduction in any medium provided the original work is properly cited. 